\section{Depression linked to poor self-care in patients with diabetes mellitus}

A new study suggests that depression might be associated with nonadherence to treatment in patients with diabetes mellitus.

Gonzalez and colleagues performed a metaanalysis of 47 studies published since 1950 that aimed to evaluate the relationship between depression and self-care behaviors in 17,319 patients with diabetes mellitus. Variables assessed included type of self-care regimen, type of diabetes mellitus, life stage, research design, level of treatment adherence, use of validated versus nonvalidated measures for depression, and use of objective versus nonobjective measures for self-care. Treatment nonadherence was broadly defined as poor adherence to medication, diet, physical exercise, glucose testing, foot examination, or scheduled medical appointments.

The authors found that depression significantly correlated with poor self-care $(P<0.0001)$. The weighted effect size of this association was in the moderate range $(r=0.21,95 \% \mathrm{Cl}$ $0.17-0.25)$. The observed association between depression and self-care varied by care regimen: missed medical appointments had the greatest effect $(r=0.31,95 \% \mathrm{Cl} 0.29-0.34)$ and foot examination had the smallest $(r=0.07$, $95 \% \mathrm{Cl}-0.08-0.21)$. Potential moderators of the association between depression and selfcare included diet, medication adherence and glucose testing; however, the relationship was unaffected by type of diabetes mellitus.

The presence of concomitant depression can worsen the outcomes of patients with diabetes mellitus. Gonzalez et al. conclude that poor selfcare behaviors might represent an important link between these two conditions.

Original article Gonzalez JS et al. (2008) Depression and diabetes treatment nonadherence: a meta-analysis. Diabetes Care 31: 2398-2403

\section{New antiobesity drug, tesofensine, shows good phase II results}

Weight loss of $5-10 \%$ is associated with improved health in obese individuals; however, currently approved antiobesity drugs typically achieve $<5 \mathrm{~kg}$ weight loss after 1 year. A double-blind, randomized, controlled, prospective trial by Astrup et al. has demonstrated that tesofensine effectively induces weight loss and improves quality of life in obese individuals.

All 203 participants were placed on an energy-restricted diet. After a 2-week run-in period, participants were randomly allocated to receive $0.25 \mathrm{mg}, 0.5 \mathrm{mg}$ or $1.0 \mathrm{mg}$ tesofensine or placebo per day for 24 weeks. In the 161 patients who completed the study, significant, mean, placebo-adjusted weight losses of $4.7 \%$, $9.2 \%$ and $10.4 \%$ were achieved in those receiving $0.25 \mathrm{mg}, 0.5 \mathrm{mg}$ or $1.0 \mathrm{mg}$ of tesofensine, respectively. A similar outcome was achieved in the intention-to-treat population. The reduction in body weight was associated with a significant improvement in weight-related quality of life.

Although the weight loss achieved by the two highest doses was similar, the treatmentrelated adverse effects were more frequent with the $1.0 \mathrm{mg}$ dose. The most common adverse effects associated with tesofensine were gastroenterological (dry mouth, nausea, constipation and diarrhea). Heart rate showed a dosedependent increase in all three groups, up to 8.1 beats per minute.

The findings from this study suggest that $0.5 \mathrm{mg}$ of tesofensine per day safely and effectively induces weight loss in obese patients. These promising findings now require validation and direct comparison with approved weight-loss drugs in large, phase III trials.

Original article Astrup A et al. (2008) Effect of tesofensine on bodyweight loss, body composition, and quality of life in obese patients: a randomised, double-blind, placebocontrolled trial. Lancet 372: 1906-1913 